\title{
Mendelian susceptibility to mycobacterial diseases
}

INSERM

\section{Source}

INSERM. (1999). Orphanet: an online rare disease and orphan drug data base. Mendelian susceptibility to mycobacterial diseases. ORPHA:748

Mendelian susceptibility to mycobacterial diseases (MSMD) is a rare immunodeficiency syndrome, characterized by a narrow vulnerability to poorly virulent mycobacteria, such as bacillus Calmette-Guérin (BCG) vaccines and environmental mycobacteria (EM), and defined by severe, recurrent infections, either disseminated or localized. 